\title{
OS DESAFIOS PARA A INTERVENÇÃO EM SAÚDE NA ESTRATÉGIA DE SAÚDE DA FAMÍLIA SEGUNDO OS PROFISSIONAIS
}

Giordano Pedro de Oliveira ${ }^{1}$, Aida Maris Peres ${ }^{2}$, Maria Marta Nolasco Chaves ${ }^{3}$, Elizabete Araújo Eduardo ${ }^{4}$

\begin{abstract}
RESUMO: Este estudo objetivou identificar facilidades e dificuldades apontadas pelos profissionais da Estratégia de Saúde da Família para o enfrentamento dos problemas de saúde das famílias e estabelecer a relação desta prática com os currículos de suas graduações. Pesquisa descritiva de abordagem qualitativa com doze profissionais de três unidades de Saúde da Família, de abril a maio de 2008, que responderam questões sobre o modelo de atenção à saúde e sua prática diária. Os resultados apontaram que o egresso não está preparado para enfrentar as contradições da realidade que se impõem no cotidiano profissional e necessita de fundamentação teórica consistente que subsidie o processo de trabalho na Estratégia Saúde da Família. Concluiu-se que a formação profissional deveria ser baseada em teorias que, junto a aproximações mais concretas da condição de vida da população, permitisse-lhes realizar reflexões críticas e compreender a realidade nas diferentes dimensões e contradições, para assim propor intervenções transformadoras.

PALAVRAS-CHAVE: Programa saúde da família; Recursos humanos em saúde; Sistema único de saúde.

\section{CHALLENGES FACED BY HEALTHCARE INTERVENTIONS IN THE FAMILY HEALTH STRATEGY ACCORDING TO HEATHCARE PROFESSIONALS}

\begin{abstract}
This study aimed to identify easy and difficult issues noted by Family Health Strategy healthcare professionals, concerning the confrontation of family health problems and establishing how that relates to the curriculums of the degree courses. This descriptive research with a qualitative approach was carried out with the collaboration of twelve healthcare professionals from three Family Health units, between April and May 2008, who answered questions about how they provided healthcare in daily practice. The results indicated that, firstly, the graduate is not prepared to confront the contradictions of reality which impose themselves on the professional routine and that, secondly, graduates need consistent theoretical grounding to support the work processes of the Family Health Strategy. It was concluded that professional training should be based in theories which, together with more concrete familiarity with the conditions of life of the population, should permit them to carry out critical reflection and understand reality in its different dimensions and contradictions, so as to offer transformative interventions.

KEYWORDS: Family health program; Human resources in health; Unified health system.

\section{LOS DESAFÍOS PARA LA INTERVENCIÓN EN SALUD EN LA ESTRATEGIA DE SALUD DE LA FAMILIA DE ACUERDO A LOS PROFESIONALES}

RESUMEN: Este estudio tuvo el objetivo de identificar facilidades y dificultades apuntadas por los profesionales de la Estrategia de Salud de la Família para el afrontamiento de los problemas de salud de las familias y establecer la relación de esta práctica con los currículos de sus graduaciones. Investigación descriptiva de abordaje cualitativo con doce profesionales de tres unidades de Salud de la Familia, de abril a mayo de 2008, que contestaron a cuestiones sobre el modelo de atención a la salud y su práctica diaria. Los resultados apuntaron que el egreso no está listo para afrontar las contradicciones de la realidad que se imponen en el cotidiano profesional y necesita de fundamentación teórica consistente para subsidiar el proceso de trabajo en la Estrategia Salud de la Familia. Se concluyó que la formación profesional debería ser basada en teorías que, junto a aproximaciones más concretas de la condición de vida de la población, posibilitase realizar reflexiones críticas y comprender la realidad en las diferentes dimensiones y contradicciones, para de ese modo proponer intervenciones transformadoras. PALABRAS-CLAVE: Estrategia salud de la família; Recursos humanos en salud; Sistema único de salud.

${ }^{1}$ Enfermeiro da Unidade de Terapia Intensiva Pediátrica do Hospital Infantil Waldemar Monastier - HI. Membro do Grupo de Pesquisa em Políticas, Gestão e Práticas em Saúde - GPPGPS da Universidade Federal do Paraná - UFPR.

${ }^{2}$ Enfermeira. Doutora em Enfermagem. Professora do Departamento e do Programa de Pós-Graduação em Enfermagem da UFPR DENF PPGENF UFPR. Vice-líder do GPPGPS.

${ }^{3}$ Enfermeira. Doutora em Enfermagem. Professora do DENF PPGENF UFPR. Vice-líder do Grupo de Pesquisa Saúde, Família e Desenvolvimento. ${ }^{4}$ Enfermeira. Coordenadora do Núcleo de Estudo, Ensino e Pesquisa do HI. Mestranda pelo PPGENF UFPR. Bolsista CAPES. Membro do GPPGPS.

Autor Correspondente:

Elizabete Araújo Eduardo

Universidade Federal do Paraná

Av. Presidente Affonso Camargo, 2305 - 80050-370 - Curitiba-PR-Brasil

E-mail: beteokale@hotmail.com
Recebido: $12 / 06 / 11$

Aprovado: $15 / 08 / 11$

Cogitare Enferm. 2011 Jul/Set; 16(3):448-54 


\section{INTRODUÇÃO}

Um sistema de saúde igualitário, descentralizado, universal e integral foi o sonho dos sanitaristas brasileiros e de diversos setores da sociedade, principalmente nas décadas de 1970 e 1980 . Esse sistema deveria ter sua organização orientada pelos dados epidemiológicos, e discutir a doença e a saúde como um processo interrelacionado aos outros processos que definem a vida das pessoas em uma determinada sociedade, em um tempo histórico.

A Estratégia Saúde da Família (ESF) surgiu no contexto de mudanças do modelo de atenção à saúde, evidenciados a partir da promulgação da Constituição Brasileira de 1988, apontando a necessidade de reorganização dos serviços do setor. Logo, a estratégia foi implantada com o propósito de atender os princípios do Sistema Único de Saúde (SUS) e fortalecer o modelo de atenção à saúde preconizado. Dados mostraram que a meta de expansão das equipes ESF, para março de 2009, era de 30 mil equipes distribuídas entre 5.251 municípios, cobrindo aproximadamente 96,1 milhões de brasileiros e com isso registrando-se um avanço de cobertura da atenção básica à saúde da população-alvo ${ }^{(1)}$.

Assim, os profissionais de saúde se defrontaram com a necessidade de adotar novas bases teóricas para nortear suas intervenções e, acredita-se que o aporte teórico-prático aprendido por estes profissionais em suas graduações se tornou determinante no êxito dessa Estratégia.

Com esta perspectiva de enfrentamento do processo saúde-doença do indivíduo ou coletividades, cabe à equipe de ESF estar preparada para executar ações administrativas, de assistência, de comunicação e de educação, que compreendam os três níveis de atenção à saúde - promoção, proteção e recuperação - tendo como caráter prioritário a prevenção de adoecimentos $^{(2)}$. Entretanto, as equipes da ESF se defrontam com várias dificuldades na sua atuação. Tais dificuldades são decorrentes da formação insuficiente dos profissionais na área da Saúde Coletiva, a qual tem sido baseada no modelo universitário tradicional que tem como foco a atenção curativo-individual e ênfase no fenômeno biológico, o que desconsidera os processos socio-psico-comportamentais implicados na doença ${ }^{(3)}$.

Somado a esta questão tem-se o desafio do trabalho em uma equipe multiprofissional e o pressuposto de que esta equipe conheça as famílias e comunidade de um determinado território. No reconhecimento do território é necessário identificar os problemas, as situações de risco à saúde e as situações de saúde indesejadas nas famílias e na comunidade. Este processo de reconhecimento é a base para planejar as intervenções que modificariam a realidade indesejada, rompendo-se com o modelo biomédico-curativo-medicalizado, conforme descrito anteriormente. Porém, acredita-se que os profissionais de saúde não tem sido preparados para o novo modelo de atenção à saúde exigido pelo atual sistema, surgindo questionamentos sobre a formação dos profissionais para atuarem neste contexto ${ }^{(4)}$.

Deste modo, o cenário apresentado indica que a formação inadequada de profissionais para atuar na equipe da ESF pode fragilizar a implantação do modelo de atenção com enfoque na promoção da saúde e prevenção de adoecimentos. Nesse sentido, os objetivos deste estudo foram: identificar as facilidades e dificuldades apontadas pelos profissionais de saúde atuantes na ESF para o enfrentamento dos problemas de saúde das famílias e comunidades assistidas e, estabelecer a relação da prática sanitária com a formação destes profissionais.

\section{METODOLOGIA}

Trata-se de uma pesquisa descritiva, de caráter qualitativo, realizada em três unidades de ESF, no Município de Curitiba-PR, no período de abril a maio de 2008. Os sujeitos da pesquisa foram doze profissionais de saúde das unidades referidas. Foram critérios de inclusão: ser enfermeiro, médico ou cirurgião-dentista trabalhando em equipe de uma unidade de saúde com ESF e que aceitasse participar da entrevista.

Os aspectos éticos relacionados à pesquisa com seres humanos foram respeitados, sendo o projeto analisado e aprovado pelo Comitê de Ética em Pesquisa do Setor de Ciências da Saúde da Universidade Federal do Paraná, processo n. 0007.0.091.000-08, e pelo Comitê de Ética em Pesquisa da Secretaria Municipal de Curitiba, sob o protocolo n. 49/2008.

Os sujeitos da pesquisa formalizaram seu consentimento por meio do Termo de Consentimento Livre e Esclarecido e para garantir os princípios do sigilo e anonimato eles foram identificados por códigos de P1 a P12. Na coleta dos dados foi utilizado um roteiro de entrevista semiestruturada enfocando tópicos referentes à compreensão do entrevistado sobre o modelo de saúde, saúde da família, facilidades e dificuldades para o exercício da prática profissional e sugestões para o ensino de graduação complementar à temática Saúde da Família.

Os dados foram analisados pela técnica de análise de conteúdo ${ }^{(5)}$ em três etapas: pré-análise, descrição analítica e interpretação referencial. Na pré-análise 
foi realizada a transcrição das entrevistas e a organização das mesmas. Em seguida, na descrição analítica, iniciou-se com a análise de conteúdo das entrevistas, para identificar as categorias emergentes e com posterior identificação dos núcleos de sentido, estabelecendo os fragmentos dos textos e nestes percebendo as convergências e divergências existentes. $\mathrm{Na}$ fase da interpretação referencial aproximou-se as categorias do material selecionado com os referênciais teóricos, no sentido de aprofundar a fase da análise já descrita.

\section{RESULTADOS}

A partir da análise temática das entrevistas, obtiveram-se as categorias e subcategorias que constam no quadro 1 .

Quadro 1-Categorias e subcategorias que emergiram do discurso dos sujeitos da equipe da Estratégia Saúde da Família. Curitiba, 2008

\begin{tabular}{ll}
\hline Categorias Empíricas & Subcategorias Empíricas \\
\hline $\begin{array}{l}\text { Facilidades do trabalho } \\
\text { na ESF }\end{array}$ & $\begin{array}{l}\text { Aproximação com o usuá- } \\
\text { Trabalia e comunidade } \\
\text { Resolutividade }\end{array}$ \\
\hline Dificuldades do trabalho & $\begin{array}{l}\text { Falta de capacitação dos re- } \\
\text { cursos humanos em saúde } \\
\text { na ESF }\end{array}$ \\
$\begin{array}{l}\text { Cobranças porprodutividade } \\
\text { Reconhecimento dos deter- } \\
\text { minantes sociais da saúde }\end{array}$ \\
\hline Formação para a ESF & $\begin{array}{l}\text { Vivência na ESF durante } \\
\text { a formação } \\
\text { Mudança de paradigma de } \\
\text { modelo de atenção à saúde }\end{array}$ \\
\hline
\end{tabular}

As falas, a seguir, apontam para os aspectos relacionados à pergunta norteadora do estudo. As facilidades e dificuldades no enfrentamento dos problemas de saúde das famílias foram evidenciadas a partir das concepções sobre Saúde da Família, destacadas dos discursos dos seguintes sujeitos:

A Saúde da Família trabalha com a família no domicílio, abrangendo questões sociais e a inserção do indivíduo na comunidade. (P1)

É um atendimento à família em vários aspectos: social, econômico [...] global. Forma vínculos com o usuário. Não atende somente a doença. (P2)
Três subcategorias foram definidas a partir da categoria Facilidades de trabalho na ESF, sendo elas: aproximação com o usuário, família e comunidade; trabalho interdisciplinar; resolutividade.

Na subcategoria "aproximação com o usuário, família e comunidade", os sujeitos citam como facilitadores do trabalho na ESF a aproximação do profissional de saúde com a realidade do usuário, assim como a organização destes em associações ou conselho local de saúde:

Uma facilidade é a participação mais efetiva da comunidade; apoio do conselho local de saúde, associação de moradores e dos equipamentos sociais locais. (P1)

Trabalhar com o usuário. A prevenção é feita dentro da realidade do usuário. Assim é menos utópica e mais concreta. (P5)

Como subcategoria, o "trabalho interdisciplinar" apareceu enquanto fator contribuinte no processo de trabalho do profissional de saúde:

A equipe multiprofissional ajuda nas dificuldades. (P4)

Trabalho em equipe. A organização das atribuições da equipe. Assim não sobrecarrega o profissional. (P9)

A resolutividade dos serviços de saúde foi citada como uma maneira de se avaliar os serviços de saúde a partir dos resultados obtidos no atendimento ao usuário:

Facilidade é a atenção que procura trabalhar com resolutividade, com vínculo e envolver a comunidade no serviço. (P11)

Na categoria Dificuldade do trabalho na ESF foram identificadas as subcategorias: dificuldade estrutural; e, trabalhar com determinantes sociais da saúde. As dificuldades estruturais foram, primeiramente, representada pela "falta de capacitação dos recursos humanos em saúde", como apontam os seguintes trechos das entrevistas:

Falta educação continuada. (P10)

Muitos funcionários vieram trabalhar pelo dinheiro e não têm perfil para a Saúde da Família. (P3)

Os profissionais entrevistados apontam como fator negativo para o desenvolvimento do trabalho 
a cobrança por produtividade, conforme consta nos trechos a seguir:

Há uma cobrança de produtividade cada vez maior. (P4)

A cobrança pela produtividade preza pela parte curativa e há pouco espaço para realizar outras atividades da Estratégia. (P2)

Destaca-se, ainda, a necessidade de reconhecimento dos determinantes sociais da saúde, que foi apontada pelos sujeitos como dificultador do trabalho na ESF:

Há dificuldades no entendimento dos usuários. Há muito analfabetismo e semi-alfabetização. (P3)

Uma dificuldade é a baixa escolaridade da população atendida. (P12)

Como dificuldade no processo, também foi citada pelos profissionais a falta de governabilidade sobre as situações sociais:

Quando se aproxima da família, vê a complexidade dos problemas. Há um sentimento de impotência! São problemas sociais, de desemprego. Há um conflito: o mesmo vínculo que facilita, também dificulta. O serviço não consegue dar a resposta. $(\mathrm{P} 11)$

No categoria Formação para a ESF, sob a ótica de como a produção pode contribuir para a atuação, as subcategorias encontradas foram: vivência na ESF durante a formação e mudança de paradigma de modelo de atenção à saúde.

No discurso abaixo, o profissional ressalta a importância da vivência da ESF durante a graduação.

Devem aumentar os espaços para colocar o aluno diretamente no PSF. Assim, na graduação, o aluno tem um contato maior com o PSF, o que ajuda na percepção da realidade. $(\mathrm{P} 1)$

A necessidade de mudança na estrutura dos cursos de graduação na área da saúde é abordada pelos profissionais entrevistados:

Na minha graduação não houve ênfase para a Saúde da Familia. Deve existir uma cadeira para saúde da família. (P12)

A formação não prepara para a realidade brasileira. (P6)

\section{DISCUSSÃO}

De acordo com os relatos, as falas dos sujeitos foram categorizadas. Teceu-se em seguida, um paralelo entre a fala dos sujeitos, a literatura e a visão dos autores de acordo com cada categoria.

\section{Aproximação com usuário, família e comunidade}

As ações da ESF, quando consideram o meio onde o indivíduo está inserido e as relações que existem dentro e fora do ambiente familiar, permitem uma compreensão ampliada do processo saúde-doença, que vai interferir no cuidar deste indivíduo. A visão deste como ser social trará maior significância para as intervenções da equipe ${ }^{(6)}$.

A inserção do indivíduo no mundo do trabalho define sua alocação em um determinado grupo social. Realizar um atendimento integral a este indivíduo significa perceber a heterogeneidade entre os usuários, determinada pelas suas características sociais ${ }^{(7)}$.

No conteúdo das entrevistas percebe-se que o processo de trabalho na ESF propicia aproximação do profissional com a realidade do usuário, conhecendo seu papel na família e sua inserção na sociedade.

Alguns participantes relataram a necessidade de incluir o usuário no planejamento da assistência à saúde, onde o enfoque seja a valorização da cultura, das condições de vida e saberes, em um gradativo processo de humanização e horizontalização da relação do profissional de saúde com o usuário( ${ }^{(8)}$.

Os equipamentos sociais no território, neste contexo, foram apontados como um dos facilitadores da prática profissional. Estas instituições podem ter papel preponderante na intervenção do profissional de saúde no processo saúde-doença do usuário. Entende-se que estes equipamentos podem oferecer à comunidade meios para ajudá-la na conquista da autonomia e exercício da cidadania. Durante a formação na graduação é relevante que o futuro profissional vivencie situações de intervenção em saúde que contemplem as parcerias com outros setores.

\section{Trabalho interdisciplinar}

O sistema público de saúde, historicamente, esteve centrado no modelo hospitalocêntrico, no qual os demais profissionais de saúde eram considerados coadjuvantes da prática médica. Contrapondo-se a este modelo, a ESF constitui-se de equipes multiprofissionais que 
devem atuar em uma perspectiva interdisciplinar. Os membros da equipe articulam suas práticas e saberes no enfrentamento de cada situação indesejada para a saúde, afim de propor soluções conjuntas e intervir de maneira adequada, já que todos conhecem a problemática ${ }^{(9)}$.

O trabalho em saúde na ESF é interdisciplinar. Ao considerar que o indivíduo expressa as diferentes dimensões da realidade - singular, particular e estrutural - a intervenção monopolizada por apenas um profissional de saúde compromete que estas sejam exploradas e, consequentemente, reduz a intervenção ao adoecimento expresso apenas na dimensão singular.

A constatação de que o trabalho interdisciplinar é importante para a operacionalização do serviço mostra que o trabalho em saúde com a ESF necessita de uma abordagem que supere as anteriores, sem interdependência com os conhecimentos dos outros profissionais da equipe.

Nesse sentido, a abordagem interdisciplinar na formação tem que ser amplamente discutida. Embora as diretrizes curriculares do Ministério da Educação apontem para a formação com a interdisciplinaridade, não é o que tem ocorrido na prática. Os egressos dos cursos da área da saúde se deparam com uma proposta metodológica para o trabalho que não foi abordada na sua graduação. A falta de compreensão do trabalho interdisciplinar gera tensões na prática, dificultando o desempenho das equipes na operacionalização dos pressupostos da ESF.

\section{Resolutividade}

A intervenção com resolutividade, conforme apontam os discursos, é compreendida como a eficiência, a capacidade de resolução das ações e dos serviços de saúde, por meio da assistência integral, contínua e de boa qualidade aos usuários e suas famílias, seja esta feita no domicílio ou na unidade de saúde. São ações que buscam identificar as causas e os fatores de risco a que essa população possa estar exposta, para então modificá-los ${ }^{(10)}$.

A capacidade resolutiva quantitativa depende do número e da distribuição de horas-trabalho dos profissionais para absorver a demanda onde e quando ela ocorre. É avaliada pelo número de usuários atendidos sobre o número de usuários que solicitaram o serviço. $\mathrm{O}$ aspecto qualitativo da demanda depende da natureza e da complexidade dos recursos ${ }^{(11)}$.

Desta forma, a resolutividade pode, também, ser avaliada como capacidade resolutiva quantitativa e qualitativa. Infere-se ainda, que a palavra utopia, uti- lizada por um dos profissionais, corresponde às ações de saúde que são descontextualizadas da realidade do usuário. Se houver esta descontextualização, o trabalho se dará de forma ineficaz, portanto, não resolutiva.

\section{Falta de capacitação dos recursos humanos em saúde}

Em um estudo feito nos documentos oficiais dos programas de qualificação de profissionais de saúde, que foram implementados na década de 1990, evidenciou-se a falta de articulação entre os setores da educação e da saúde, o que também ocorreu na formação profissional daquele perío$\mathrm{do}^{(12)}$. Essa desarticulação contraria as propostas dos documentos que advogavam que o processo de educação devia estar articulado com todos os níveis de organização do SUS e ser desenvolvido a partir da problematização das realidades dos sujeitos envolvidos, segundo o relatório da $8^{\text {a }}$ Conferência Nacional de Saúde ${ }^{(13)}$.

A formação de profissionais de saúde, nos últimos anos, teve um grande avanço quantitativo, porém, apresentam-se contradições nos aspectos qualitativos, e entre essas destaca-se a fundamentação teórica baseada nas concepções biologicistas com enfoque na doença e na medicalização, que não discute a determinação social do processo saúde-doença ${ }^{(12)}$.

\section{Cobrança por produtividade}

O município recompensa o desempenho das equipes baseado na Gratificação de Incentivo ao Desenvolvimento de Qualidade dos Serviços - IDQ. Este é considerado fator motivacional para o gestor, que gratifica diferenciadamente os funcionários que alcançam as metas do POA (Plano Operativo Anual), porém, é visto pelos sujeitos deste estudo como fator de desgaste e contraditório à proposta de promoção da saúde e prevenção de doenças.

\section{Reconhecimento dos determinantes sociais da saúde}

A visão integral do indivíduo e o contato com o cotidiano do usuário indicam a indissociabilidade dos determinantes sociais da situação de saúde.

A baixa escolaridade é um fator que agrava a desigualdade social. A educação de baixa qualidade tem o impacto de perpetuação do ciclo de pobreza entre gerações, uma vezque os pais com baixa escolaridade têm dificuldade em 
garantir um maior nível de escolaridade para seus filhos ${ }^{(14)}$.

A pobreza não pode ser definida de forma única, mas ela se evidencia quando parte da população não é capaz de gerar renda suficiente para ter acesso sustentável aos recursos básicos que garantam uma qualidade de vida digna. Estes recursos são água, saúde, educação, alimentação, moradia, renda e cidadania $^{(14)}$.

Os profissionais entrevistados colocam que os problemas sociais - principalmente a pobreza - vão além da capacidade de resposta da equipe. É necessário que se tenha uma compreensão dos problemas sociais como uma questão que transcende a realidade local. Sendo assim, o profissional de saúde que atua na ESF tem que compreender que o Brasil sofre um processo de reestruturação produtiva que tem significado a acentuação das desigualdades sociais, em termos de classe e de região. Tal processo, nas últimas décadas, tem aumentado a concentração de renda e, consequentemente, a pobreza. Nos últimos anos, alguns programas assistenciais têm sido utilizados para amenizar essa exclusão social, mas não há propostas que avancem nas mudanças estruturais que, de fato, modificariam essa realidade.

O processo de formação de profissionais de saúde precisa rever o atual enfoque e buscar o desenvolvimento de competências profissionais em dimensões que extrapolem o âmbito técnico-científico, ampliando a capacidade de compreensão política sobre a realidade de vida da população, e assim, potencializar a atuação dos profissionais de saúde para além do atendimento apenas aos sinais e sintomas dos adoecimentos. Diante da sua incapacidade em compreender os processos que determinam as realidades de saúde dos usuários do serviço, o profissional de saúde sente-se impotente e desmotivado.

\section{Vivência na ESF durante a formação}

Atividades complementares, em um percentual de $5 \%$ da carga horária total da formação acadêmica sugerida pelas Diretrizes Curriculares Nacionais, reforçam a necessidade de aumentar as oportunidades de, durante a graduação, os sujeitos participarem de atividades extracurriclares ${ }^{(15)}$.

Cabe à universidade reorientar a grade curricular para dar oportunidade ao aluno de ter maior contato com a ESF, por meio de aulas práticas, estágios e atividades de pesquisa e extensão, nos diferentes cursos da área da saúde.

\section{Mudança de paradigma de modelo de atenção à saúde}

Quanto à formação para a ESF, as preocupações a este respeito estão refletidas nos relatórios das Conferências Nacionais de Recursos Humanos de 1986 e 1993, assim como na Norma Operacional Básica de Recursos Humanos para o SUS (NOB/RH/ SUS-2002) ${ }^{(16)}$, documentos que refletem a posição de um conjunto representativo de segmentos e atores sociais em torno deste tema.

A edição das novas Diretrizes Curriculares para os cursos da área da saúde pelo MEC, em 2001, veio com a finalidade de corrigir essas deficiências na formação dos profissionais, eliminando a ênfase nas especializações e procurando formar um novo ator social: o profissional com formação generalista, direcionado primordialmente à atenção básica de saúde e capaz de resolver a maior parte dos problemas de uma população que tem dificuldades para atender às suas próprias necessidades, ou seja, garantir sua sobrevivência ${ }^{(17)}$.

Os trabalhadores atuantes na ESF expressam que é necessário reorientar o modelo de atenção à saúde para adequá-lo à realidade. Afirmam que a atual formação nos cursos da área da saúde não atende às especificidades do SUS. Ressaltam também que a formação precisa desenvolver conteúdos e práticas relacionados à promoção da saúde e prevenção de adoecimentos, ser adequada à realidade brasileira, valorizar a intervenção na atenção básica com ESF e priorizar a Saúde Coletiva.

\section{CONCLUSÃO}

A fundamentação teórica para a prática sanitária na ESF não foi expressa por muitos dos entrevistados. Pode-se afirmar que tal desconhecimento se deve à desarticulação entre o processo de formação e os serviços de saúde, que expressa um distanciamento entre a prática e a teoria.

Este distanciamento se torna mais acentuado quando o profissional de saúde não consegue articular o conhecimento advindo da sua formação com a prática que está desenvolvendo. Em muito dos casos, a teoria foi vista como algo idealizado e que na imposição da realidade do trabalho não é revisitada pelos profissionais de saúde, pois não a compreendem como uma ferramenta para a intervenção que desenvolvem.

O profissional de saúde que trabalha junto ao contexto familiar do usuário se defronta com questões que vão além do que foi abordado na sua graduação. Essas são questões que determinam aquela realidade, mas estão distantes da mesma. No caso, se fala da dimensão estrutural - políticas 
econômicas e sociais - mas que na formação tecnicista não possibilita compreender, assim como refletir qual é o enfrentamento possível para a transformação da realidade, a partir do horizonte em que se encontra. Com tais desafios postos, há que se comprometer a formação com referenciais críticos e reflexivos sobre a determinação social do processo saúde-doença.

Cabe ressaltar que não é apenas o profissional de saúde que define o processo de mudança no modelo de atenção à saúde. Ao contrário, para que ocorra esta mudança deve ser incluída uma complexa lista de fatores interdependentes e conflituosos, na dimensão estrutural (ideológicos e políticos), que influenciam e definem as políticas públicas de saúde e de educação. São processos distantes das dimensões singular e particular, mas definidores de como viverão os indivíduos nas suas comunidades, assim como definidores do modelo de intervenção que será referendado nos serviços de saúde.

Diante do resultado deste estudo afirmamos que, mesmo que se tenha avançado na expansão e consolidação da ESF, ainda persiste uma formação ideológica para uma prática biologicista nas graduações da área da saúde, que não prepara os futuros profissionais para enfrentar a realidade de famílias e comunidades de maneira a transformá-la para promover a saúde.

\section{REFERÊNCIAS}

1. Ministério da Saúde (BR). Departamento de Atenção Básica [Internet]. Brasília: MS; [acesso em 03 jun 2011] Atenção básica e a saúde da família. Disponível: http:// dab.saude.gov.br/abnumeros.php

2. Ministério da Saúde (BR). [Internet]. Brasília: MS; [acesso em 03 jun 2011]. Norma Operacional Básica do Sistema Único de Saúde 01/96: gestão plena com responsabilidadade pela saúde do cidadão. Nov 1996. Disponível: http://portal. saude.gov.br/portal/arquivos/pdf/nob96.pdf

3. Ferreira TSA, Campos LF. O conhecimento veiculado em literatura nacional sobre integralidade em saúde. Cogitare Enferm. 2009;14(2):368-73.

4. Pires ROM. Complexidade em saúde da família e formação do futuro profissional de saúde. Interface Comun Saúde Educ. 2009;13(30):153-66.

5. Bardin L. Análise de conteúdo. Lisboa: Persona Edições; 1977.

6. Santos MR, Ribeiro LCC. Percepção do usuário da estratégia da saúde da família sobre a função da enfermeira. Cogitare Enferm. 2010;15(4):709-15.
7. Campos CMS, Mishima SM. Necessidades de saúde pela voz da sociedade civil e do Estado. Cad Saude Publica. 2005;21(4):1260-8.

8. Ministério da Saúde (BR). Saúde da família: uma estratégia para a reorientação do modelo assistencial. Brasília: DAB; 1997.

9. Oliveira EM, Spiri WC. Programa saúde da família: a experiência de equipe multiprofissional. Rev Saúde Pública. 2006;40(4):727-33.

10. Ministério da Saúde (BR). Marco legal: saúde, um direito dos adolescentes. Brasília: Ministério da Saúde; 2005.

11. Turrini RNT, Lebrão ML, Cesar CLG. Resolutividade dos serviços de saúde por inquérito domiciliar: percepção do usuário. Cad Saude Publica. 2008;24(3):663-4.

12. Chaves MMN, Oliveira GP. As políticas públicas para a formação dos profissionais de saúde nos anos 90: uma contribuição para o debate das práticas sanitárias do Sistema Único de Saúde. Out p. 427. Curitiba: UFPR, 2007.

13. Ministério da Saúde (BR). $8^{a}$ Conferência Nacional de Saúde. Brasília: Ministério da Saúde; 1987. Relatório final. $29 \mathrm{p}$.

14. Gomes MA, Pereira MLD. Família em situação de vulnerabilidade social: uma questão de políticas públicas. Cienc Saude Colet. 2005;10(2):357-63.

15. Medeiros VC, Peres AM. Atividades de formação do enfermeiro no âmbito da atenção básica à saúde. [apresentação ao Congresso Internacional de Pesquisa em Enfermagem; 23 out 2010; Florianópolis, Brasil.

16. Ministério da Saúde (BR). Norma Operacional Básica para NOB/RH-SUS. Brasília; 2002.

17. Santos MAM, Cutolo LRA. A interdisciplinaridade e o trabalho em equipe no programa saúde da família. Arq Catarin Med. 2004;33(3):31-40. 